\title{
Mecanismos regulatorios del tono vascular pulmonar neonatal. Una perspectiva molecular
}

\author{
FELIPE A. BEÑALDO F.*, JAVIERA C. FERRADA D.*, \\ SEBASTIÁN CASTILLO G.* y GERMÁN EBENSPERGER D.*
}

\section{Regulatory Mechanisms of neonatal pulmonary vascular tone. A molecular perspective}

The extrauterine-milieu adaptation includes a considerable increase in $\mathrm{PaO}_{2}$, that specifically induces structural and vasoactive changes at pulmonary circulation. Such changes transform a poor irrigated circulation into a circulation that receive $\sim 100 \%$ of neonatal cardiac output, supporting the normal alveolar-capillary gas exchange. Local regulation of basal neonatal pulmonary circulation is maintaining by a delicate equilibrium between vasoconstrictor and vasodilator agents. This equilibrium, allows to maintain the pulmonary circulation as an hemodynamic system with a high blood flow and a low vascular resistance. Vasocontrictors action allows actin and light-chain myosin interaction. Two main pathways induced this effect in smooth muscle cell: a) a calcium dependent pathway, that increases intracellular calcium, facilitating actin - myosin binding, and b) the independent calcium pathway, which achieves through consecutive phosphorylation reactions sensitize the proteins involved, promoting the binding of actin and light-chain myosin. These actions are mediated by agonists produced mainly in the pulmonary endothelium, such as endothelin-1 and thromboxane, or by agonists from other cell types such as serotonin. Vasodilator agents regulate the vasoconstrictor response, mainly by inhibiting signals that induce calcium-independent vasoconstriction, through activation of protein kinases, which in turn will inhibit the function of ROCK kinase, one of the last effectors of vasoconstriction before formation of the actin and light-chain myosin binding. This review will focus on describing these mechanisms of primal importance in the first hours of our lives as independent individuals.

Key words: Humans; new born; pulmonary circulation; vasoconstrictor agents; vasodilator agents.

\section{Resumen}

La adaptación al medio extrauterino incluye un aumento considerable de la $\mathrm{PaO}$, que induce especialmente cambios estructurales y vasoactivos en la circulación pulmonar, que llevarán a una circulación previamente pobremente irrigada, a recibir $\sim 100 \%$ del gasto cardíaco del recién nacido, permitiendo el normal intercambio gaseoso. La regulación local de la circulación arterial pulmonar neonatal basal, es mantenida por un delicado equilibrio entre agentes vasoconstrictores y vasodilatadores. Este equilibrio, permite mantener la circulación pulmonar como un territorio de gran flujo sanguíneo y baja resistencia. La acción de los vasoconstrictores permite la formación de las interacciones entre actina y la cadena liviana de la miosina, esta es inducida en la célula muscular lisa principalmente por dos vías: a) dependiente de calcio, que consiste en aumentar el calcio intracelular, facilitando finalmente la unión de actina y miosina, y b) independiente de calcio, la cual a través de consecutivas fosforilaciones logra sensibilizar a las proteinas involucradas promoviendo la unión de actina y miosina. Estas acciones son mediadas por agonistas generados principalmente en el endotelio pulmonar, como endotelina-1 $y$ tromboxano, o por agonistas provenientes de otros tipos celulares como la serotonina. Los agentes vasodilatadores regulan la respuesta vasoconstrictora, principalmente inhibiendo la señalización que induce la vasocontricción independiente de calcio, a través de la activación de proteínas quinasas que inhibirán la función de la ROCK quinasa, uno de los últimos efectores de la vasocontricción antes de la formación de la unión de actina y miosina. Esta revisión describe estos mecanismos de primordial importancia en las primeras horas de nuestra vida como individuos independientes.

Palabras clave: Seres humanos; recién nacido; circulación pulmonar; agentes vasoconstrictores; agentes vasodilatadores.

* Unidad de Fisiología y Fisiopatología Perinatal, Programa Fisiopatología, Instituto Ciencias Biomédicas, Facultad de Medicina Campus Oriente, Universidad de Chile. 


\section{Glosario de abreviaturas utilizadas en este artículo}

5HT : Serotonina

AA : Ácido araquidónico

$\mathrm{AC} \quad$ : Adenilato ciclasa

AMPc : Adenosina-monofosfato cíclico

Arg-2 : Arginasa-2

$\mathrm{CaMq}$ : Complejo $\mathrm{Ca}^{2+}$ y calmodulina

cGMP : Guanosín monofosfato cíclico

$\mathrm{CML}$ : Células musculares lisas

CO : Monóxido de carbono

CPI-17 : C-kinase-activated protein phosphataselinhibitor

CRF : Capacidad residual funcional

DAG : Diacilglicerol

eNOS : Enzima NO sintasa endotelial

ET-1 : Endotelina-1

Gas : Proteína G estimuladora alfa ligante de guanosina

$\mathrm{H}_{2} \mathrm{O}_{2} \quad$ : Peróxido de hidrógeno

H4B : Tetrahidrobiopterina

HO : Hemoxigenasa

iNOS : Enzima NO sintasa inducible

IP3 : Inositol trifosfato

MLC : Cadena liviana de la miosina (MLC: myosin light chain)

MLCK : Cadena ligera o liviana de miosina quinasa

MLCP : Fosfatasa de la cadena liviana de la miosina

MYPT1: Myosin phosphatase targeting protein

nNOS : Enzima NO sintasa neuronal

NO : Óxido nítrico

NOS : Enzima NO sintasa

$\mathrm{PGI}_{2} \quad$ : Prostaciclina

PIP2 : Fosfatidil inositol 4,5-bifosfato

PKA : Proteína quinasa A

PKG-1 : Proteína Quinasa dependiente de cGMP

PLA2 : Fosfolipasa $\mathrm{A}_{2}$

PLB : Fosfolamban

PLC : Fosfolipasa C

QCLM : Quinasa de la cadena liviana de miosina

ROCK : Quinasa de Rho A

RYR : Canales receptores de rianodina

SERCA : Sarco/endoplasmic reticulum $\mathrm{Ca}^{2+}$-ATPase

sGC : Guanilato Ciclasa Soluble

SOCE : Store Operated Calcium Entry

STIM : Moléculas de interacción estromal

TRPC : Transient Receptor Potential Cation Channel

$\mathrm{TxA}_{2}$ : Tromboxano $\mathrm{A}_{2}$

TXS : Tromboxano sintasa

\section{Introducción}

Durante la vida intrauterina, la circulación pulmonar fetal está caracterizada por una resistencia vascular alta y un flujo sanguíneo pulmonar bajo, de tal manera que solo alrededor del $10 \%$ del gasto cardiaco combinado fluye a través de los pulmones, mientras el intercambio gaseoso de la sangre fetal se realiza en la placenta ${ }^{1}$. El bajo flujo sanguíneo pulmonar y la elevada resisten- cia vascular pulmonar fetal dependen de varios factores tales como: la alta presión extraluminal alrededor de la vasculatura pulmonar, debido a que los pulmones están llenos de líquido; un contenido relativamente bajo en sustancias vasodilatadoras, como lo son el óxido nítrico (NO) y prostaciclina $\left(\mathrm{PGI}_{2}\right)$, y particularmente por la baja presión de oxígeno $\left(\mathrm{PO}_{2} 12-15 \mathrm{mmHg}\right.$ a nivel del mar) en la circulación fetal2,3

La transición neonatal desde la vida fetal hacia los primeros días de vida está determinada por cambios fisiológicos importantes en las funciones hemodinámicas iniciadas por el trabajo de parto, la ventilación pulmonar, y el pinzado del cordón umbilical, pero muy especialmente por el aumento de la $\mathrm{PO}_{2}$ en los alvéolos y en la sangre pulmonar $^{4}$. La eliminación y absorción de líquidos pulmonares y la ventilación, son claves no solo para el establecimiento de la capacidad residual funcional (CRF), que permite evitar el colapso alveolar, sino también y especialmente para el descenso de la resistencia vascular pulmonar.

La circulación pulmonar se transforma rápidamente, de un sistema de bajo flujo y alta resistencia, a uno de alto flujo y baja resistencia, produciéndose el cierre funcional de las comunicaciones anatómicas como el ductus arteriosus $\mathrm{y}$ el foramen ovale. Estos eventos, pero muy en particular el incremento de la $\mathrm{PO}_{2}$, generan aumentos en la expresión y la actividad de mecanismos de señalización de un número de moléculas vasodilatadoras como $\mathrm{PGI}_{2}$ y $\mathrm{NO}$, y una disminución de mecanismos vasoconstrictores como endotelina-1 (ET-1) y el factor activador de plaquetas ${ }^{3,5,6}$. Conjuntamente, se produce un notable remodelamiento fisiológico de la estructura de las arterias pulmonares, cuya principal característica es el adelgazamiento de la pared arterial pulmonar, como resultado de una disminución en el número de células de músculo liso arterial pulmonar, y un aplanamiento de las células endoteliales pulmonares, entre otros mecanismos ${ }^{3,7,8}$.

El tono vascular pulmonar basal es regulado por un delicado equilibrio entre agentes vasoconstrictores (ET-1, Serotonina y tromboxano) y agentes vasodilatadores (NO, $\left.\mathrm{CO}, \mathrm{cGMP}, \mathrm{PGI}_{2}\right)$. En esta revisión, profundizaremos en los mecanismos moleculares que participan en la regulación local del tono vascular pulmonar neonatal.

\section{Aspectos básicos de la vasoconstricción.}

Se define la contracción de los vasos sanguíneos como la disminución del lumen vascular, mediante la formación del puente entre las fibras 
de actina y la cadena liviana de la miosina en las células musculares lisas (CML), y que es regulada mediante la activación de receptores ubicados en las células endoteliales o las CML. Los factores que participan en estimular la vasoconstricción pueden ser de distintas naturalezas $\mathrm{u}$ orígenes, por ejemplo, pueden ser físicos por estimulación directa de las células endoteliales (shear stress o deformación por el esfuerzo), o químicos donde se estimulan los receptores de la célula muscular lisa (serotonina, tromboxano $\mathrm{y}$ endotelina).

\section{Vasoconstricción dependiente de calcio}

La contracción de las CML depende de la fosforilación de la cadena liviana de miosina, proceso que le permitirá la interacción con los filamentos de actina ${ }^{9}$. La quinasa de la cadena liviana de miosina (QCLM) es una enzima dependiente de $\mathrm{Ca}^{2+}$ y calmodulina (formación del complejo CaMq), y esta podría activarse con cualquier estímulo que promueva un aumento de la concentración citosplamática de $\mathrm{Ca}^{2+}{ }^{(10)}$.

La activación de los receptores de agonistas pro-vasoconstrictores, inducen la activación de las proteínas $\mathrm{G}$ y estas a su vez, activan las fosfolipasas, formándose $\mathrm{PIP}_{2}$, (fosfatidil inositol 4,5-bifosfato) a partir del cual se formarán IP3 (inositol trifosfato) y DAG (diacilglicerol). El aumento en la producción de $\mathrm{IP}_{3}$, permitirá que se una a su receptor, presente en canales de calcio en el retículo sarcoplásmico, liberando este ión hacia el compartimiento intracelular. El aumento de calcio es detectado por calmodulina, formando el complejo Ca-calmodulina. Este complejo, actuará de dos maneras: 1) Activando la quinasa de la cadena liviana de la miosina (QCML), fosforilando a la cadena liviana de la miosina y generando la interacción de miosina con actina, produciendo finalmente la vasoconstricción; 2) Activando a enzimas que mantienen inhibida la acción de la miosina ATPasa en concentraciones bajas de $\mathrm{Ca}^{2+}$, por lo que se libera la inhibición de la miosina ATPasa y se facilita la generación de puentes entre actina y miosina ${ }^{10}$ (Figura 1).

En la membrana del retículo sarcoplasmático se encuentran las moléculas de interacción estromal (STIM), moléculas encargadas de detectar el vaciamiento de calcio reticular. Cuando el $\mathrm{Ca}^{2+}$ reticular es liberado por algún estímulo, STIM oligomeriza y transloca hacia las zonas punctae del retículo, permitiendo así una interacción con la membrana plasmática de la CML. Esta acción permitirá la unión física con homo o heterotertrámeros de TRPC (Transient Receptor Potential Cation Channel Subfamily C) u ORAI (término de la mitología griega que significa 'el guardador de llaves'), gatillando la entrada de $\mathrm{Ca}^{2+}$ desde el espacio extracelular hacia el intracelular, proceso conocido como "entrada de calcio operada por depósito" (SOCE, del inglés Store Operated Calcium Entry) ${ }^{11,12}$ (Figura 1).

Por otra parte, el aumento de $\mathrm{Ca}^{2+}$ intracelular participa en la activación de enzimas quinasas, a través de proteína quinasa $\mathrm{C}$, que fosforilan enzimas fosforiladoras (CPI-17), que a su vez también puede ser fosforilada por ROCK, (quinasa de RhoA) amplificando y prolongando la señalización. CPI-17 fosforila a la fosfatasa de la cadena liviana de la miosina (MLCP) en la unidad catalítica de MYPT1 (myosin phosphatase targeting protein), inhibiendo su efecto fosfatasa y generando vasoconstricción ${ }^{9,13,14}$. Otra vía de aumento de calcio intracelular son los canales de calcio sensibles a voltaje, tipo L y T. La apertura de estos canales dependerá de cambios en el potencial de membrana. Específicamente, cualquier estímulo que genere una despolarización de la membrana plasmática, permitirá la apertura de estos canales, gatillando así la entrada de calcio del espacio extracelular al intracelular ${ }^{15}$.

Los canales receptores de rianodina (RYR) se encuentran en la membrana del retículo sarcoplásmico, estos canales detectan el aumento de $\mathrm{Ca}^{2+}$ intracelular generado por los mecanismos anteriormente descritos, y liberan $\mathrm{Ca}^{2+}$ al intracelular desde el retículo sarcoplásmico, por lo que contribuyen al incrementos de $\mathrm{Ca}$ intracelular en zonas específicas de la CML y durante breves momentos ( $C a$ spark o centelleo de calcio), el mecanismo de apertura de estos canales es mediante fosforilación por quinasas (PKA), las que a su vez son activadas por proteínas G. Por último, la bomba Ca-ATPasa SERCA (sarco/endoplasmic reticulum $\mathrm{Ca}^{2+}$-ATPase), que hace que ingrese $\mathrm{Ca}^{2+}$ al interior del retículo sarcoplasmático, se encuentra inhibida por fosfolamban, manteniendo los niveles de calcio intracelular altos $^{16}$. La actividad de la SERCA2a está bajo el control de una fosfoproteína de 52 aminoácidos, asociada a la membrana del retículo sarcoplásmico, denominada fosfolamban (PLB). En su estado desfosforilado, fosfolamban inhibe a la SERCA2a y el transporte de $\mathrm{Ca}^{2+}$ hacia el retículo sarcoplásmico.

\section{Mecanismo independiente de calcio}

En condiciones de concentración de calcio intracelular constante, la vasoconstricción de la célula muscular lisa está asociada a una sensibilización al calcio, que es un importante factor en la respuesta constrictora, pues se asocia a muchos 


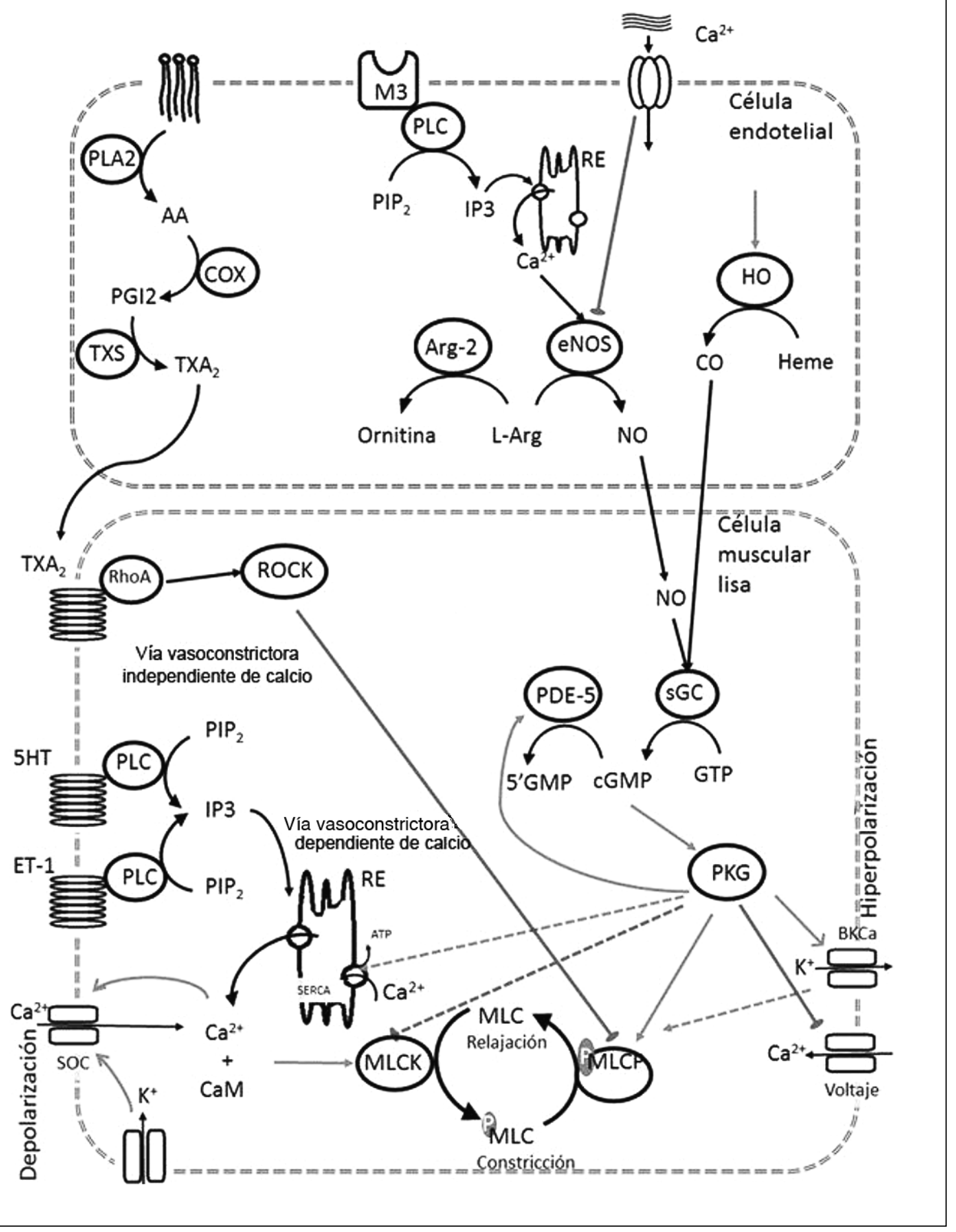

Figura 1. Esquema de las principales vías vasoconstrictoras y vasodilatadoras que gobiernan el tono vascular pulmonar neonatal (ver texto). Abreviaturas: M3: Receptor muscarínico M3; PLA: fosfolipasa A; PLC: fosfolipasa C; AA: ácido araquidónico; COX: ciclo-oxigenasa; $\mathrm{PGI}_{2}$ : prostaciclina; TXS: Tromboxano sintasa; $\mathrm{TXA}_{2}$ : Tromboxano $\mathrm{A}_{2}$; $\mathrm{PIP}_{2}$ : fosfoinositol bifosfato; $\mathrm{IP}_{3}$. Fosfoinositol trifosfato; RE: retículo endoplásmico; eNOS:NO sintasa endotelial; HO. Hemooxigenasa; L-Arg: L- arginina. 5HT: Serotonina; ET-1: endotelina 1; SOC: store operated channel; ROCK: quinasa de RhoA, CaM: calmodulina; SERCA: sarcolendoplasmic reticulum $\mathrm{Ca}^{+2}$ ATPase; MLCK: cadena ligera de miosina quinasa; MLC: cadena liviana de miosina ; MLCP: fosfatasa de la cadena liviana de miosina; PDE-5: fosfodiesterasa 5; sGC: guanil ciclasa soluble; cGMP: Guanosín Monofosfato cíclico, GTP: guanosín trifosfato; PKG: Proteína Quinasa dependiente de cGMP; BKA: Canal de potasio dependiente de calcio de gran conductancia. 
agonistas y a proteínas quinasas que median esta respuesta. Este mecanismo es comandado por la fosforilación de la cadena liviana de la miosina (MLC: myosin light chain), al existir una unión entre receptores transmembrana de agonistas vasoconstrictores ligados a proteínas $\mathrm{G}$ y distintos agentes vasoconstrictores, tales como endotelina (ET-1) y sus receptores en células musculares lisas, tromboxano $\left(\mathrm{TxA}_{2}\right)$ y Serotonina (5HT), entre otros ${ }^{3}$.

La vasoconstricción independiente de calcio, al igual que la dependiente, involucra proteínas $\mathrm{G}$, las que activan enzimas RhoA-GTP asas, que inducen la activación de las enzimas ROCK, que son una familia de serina/treonina quinasa, que participa en la fosforilación de distintas proteínas, que regulan la vasocontracción y la proliferación de las células musculares lisas pulmonares, dentro de estas proteínas, ROCK fosforila a CPI-17, que es una proteína inhibitoria endógena de la fosfatasa de la miosina. La CPI-17 se une a la unidad catalítica de MYPT1, inhibiendo a MYPT1, específicamente, fosforila a MYPT1 en dos treoninas, afectando la unidad regulatoria y por ende la desfosforilación de MLC, manteniendo la vasoconstricción ${ }^{14,17,18}$.

Los receptores, de los agonistas vasoconstrictores, de las células musculares se encuentran unidas a fosfolipasa C (PLC) o al homólogo de la familia génica Ras. Las proteínas Ras son una parte de una gran familia de GTPasas. En los seres humanos, la superfamilia Ras se divide en 6 subfamilias: Ras, Rho, Arf, Rab, Ran y Rad. En este caso se trata del miembro A (RhoA) dependiendo del agonista a unir, en el caso de la Endotelina (ET-1) los receptores son ETA y ETB y están ligados a una PLC al igual que los receptores de Serotonina (5HT) 5HT1B/D, 5HT2A y 5HT2B que están ligados a una PLC ${ }^{19,20}$ (Figura 1).

\section{Agonistas vasoconstrictores}

Los principales agentes vasoconstrictores de la circulación pulmonar neonatal son endotelina-1 (ET-1), serotonina (5-HT) y tromboxano. La endotelina-1, es un potente vasoconstrictor, de naturaleza polipéptidica, de 21 aminoácidos producido principalmente por las células endoteliales ${ }^{3}$. Además, ET-1 presenta propiedades mitogénicas e hipertróficas, a través de estimular la proliferación, migración, contracción, remodelamiento de la matriz extracelular y la secreción de factores de crecimiento y mediadores inflamatorios en las células musculares lisas ${ }^{3}$. Ejerce su efecto de una manera autocrina y paracrina a través de la activación de sus dos subtipos de receptores, ETA y ETB. Ambos receptores perte- necen a la gran familia de receptores transmembrana acoplados a proteínas G. Los receptores ETA están altamente expresados en las células musculares lisas de vasos sanguíneos, mientras que los receptores ETB son los predominantes en las células endoteliales, y ambos receptores exhiben roles fisiológicos relativamente distintos. La activación de receptores ETA por ET-1 contribuye a la vasoconstricción, estimulando ambas vías de la contracción, la dependiente y la independiente de calcio, además estimula el crecimiento y la adhesión celular, mientras que la unión de ET-1 a ETB lleva a una vasodilatación por liberación de óxido nítrico (NO) y prostaciclinas, induciendo la respuesta vasodilatadora dependiente de endotelio ${ }^{3}$.

La serotonina (5-HT), tiene un importante papel en el período fetal en la mantención de la resistencia vascular pulmonar elevada ${ }^{21}$, y posterior al nacimiento sus niveles disminuyen ${ }^{21,22}$. La respuesta celular de 5HT es a través del aumento de la actividad de la PLC acoplada a proteína $\mathrm{Gq}$, produciendo diacilglicerol e inositol 1,4,5 - trifosfato $\left(\mathrm{IP}_{3}\right)^{23}$. IP3 se une a receptores específicos del retículo endoplásmico, produciendo la liberación de calcio $\left(\mathrm{Ca}^{2+)}\right.$, aumentando la concentración del calcio citosólico $\left(\left[\mathrm{Ca}^{+2}\right] \mathrm{i}\right)$. Este aumento de $\left[\mathrm{Ca}^{2+}\right] \mathrm{i}$ junto a diacilglicerol activan a la proteína quinasa $\mathrm{C}(\mathrm{PKC})$, que fosforila proteínas específicas ${ }^{23,24}$ (Figura 1).

El tromboxano $\mathrm{A}_{2}\left(\mathrm{TxA}_{2}\right)$ es un prostanoide inestable, metabolito del ácido araquidónico $(\mathrm{AA})^{25}$. Puede ser producido en el endotelio por acción de la enzima tromboxano sintasa (TXS), y activa los receptores de tromboxano $\mathrm{A}_{2}$ en la membrana del musculo liso. Estos, se encuentran unidos a una molécula de RhoA, esta última es la que activa la Rho Quinasa (ROCK), que a su vez fosforila la MLCK (quinasa de la cadena ligera o liviana de miosina), activándola e induciendo finalmente la vasocontracción ${ }^{19,20}$. Además, la cascada de señalización mediada por tromboxano se inicia con la unión al receptor específico de membrana acoplado a proteína $\mathrm{G}$, que induce la ruptura del fosfatidilinositol 4,5 bisfosfato, vía fosfolipasa C (PLC), generando inositol trisfosfato (IP3), y continuando a través de la vía dependiente de calcio. La acción de la PLC origina también la producción de diacilglicerol (DAG), el cual activa a la proteína kinasa $\mathrm{C}(\mathrm{PKC})$. Las vías de activación mediadas por $\mathrm{Ca}^{+2}$ y por proteína quinasa (PKC) actúan de modo sinérgico, produciendo efectos en términos de activación celular y consecuentemente en la contracción del musculo liso vascular ${ }^{25}$ (Figura 1). 


\section{Aspectos básicos de la vasodilatación.}

La relajación del músculo liso permite el aumento del radio del lumen de los vasos sanguíneos. Esta relajación ocurre ya sea como resultados de la remoción del estímulo contráctil, es decir, iniciar la salida del intracelular del $\mathrm{Ca}^{2+}$, hacia el extracelular o al retículo sarcoplásmico, o por una acción directa que inhiba el mecanismo contráctil, o sea, inhibiendo la señalización que finalmente fosforilará la MYPT1, "deteniendo" así la vasoconstricción. En otras palabras, sin importar cuál de los dos sea el involucrado, el proceso de relajación requiere de una disminución del $\left[\mathrm{Ca}^{+2}\right] \mathrm{i}, \mathrm{y}$ un aumento en la actividad de la fosfatasa de la cadena liviana de miosina (MLCP).

Dentro de los agentes vasodilatadores más importantes en el período neonatal, encontramos el óxido nítrico (NO), gasotransmisor producido en el endotelio, por la enzima NO sintasa (NOS). Se conocen tres isoformas de NOS, inducible (iNOS), endotelial (eNOS) y neuronal (nNOS). La eNOS es el regulador más importante en la producción de NO en la circulación pulmonar perinatal ${ }^{3}$. La eNOS cataliza la oxidación de Larginina a L-citrulina, que involucra la activación de una molécula de oxígeno $\left(\mathrm{O}_{2}\right)$ gracias a la presencia de tetrahidrobiopterina (H4B), que al ser reducida permite la formación de $\mathrm{NO}$, este gas difunde a la vecina célula muscular lisa, donde estimula a la Guanilato Ciclasa Soluble (sGC) catalizando la producción de Guanosín Monofosfato cíclico (cGMP), el segundo mensajero predominante en la vía vasodilatadora $(28,29)$. En el endotelio vascular, la Arginasa-2 (Arg-2), compite con la NOS por la L-Arginina, y un predominio de la función arginasa, se asocia con disfunción endotelial ${ }^{26}$. Además, la función endotelial durante los primeros días de vida parecería no ser óptima, pues la respuesta a metacolina -un análogo no hidrolizable de acetilcolina- mediante la activación de los receptores muscarínicos de la célula endotelial es baja en recién nacidos sa$\operatorname{nos}^{27,28}$ (Figura 1).

Otro gasotransmisor que activa la sGC es el monóxido de carbono (CO), aunque de manera menos potente que la activación por NO. Este transmisor es sintetizado por la Hemoxigenasa $(\mathrm{HO})^{29,30}$. La HO es una enzima microsomal con tres isoformas diferentes, HO-1 es inducida frente a estímulos tales como radiación ultravioleta, peróxido de hidrógeno $\left(\mathrm{H}_{2} \mathrm{O}_{2}\right)$, hipoxia, pro-oxidantes, inflamación, metales pesados y $\mathrm{NO}^{30}$, la HO-2, está normalmente expresada las capas endoteliales y musculares de los vasos sanguíneos y generan $\mathrm{CO}$ que modula el tono vascular bajo condiciones fisiológicas, HO-3, comparte una considerable homología con HO-2, aún no tiene una función claramente descrita ${ }^{30}$ (Figura 1).

La generación de cGMP es mediada por isoformas de la enzima sGC y Guanilato Ciclasa particulada (pGC) $)^{3,31}$. La pGC es estimulada por péptidos natriuréticos ${ }^{31}$, mientras que, la $\mathrm{sGC}$ es estimulada por NO y CO. La sGC puede existir en dos formas distintas, la forma nativa o reduci$\mathrm{da}$, contenedora del grupo heme, que es la forma receptora endógena de $\mathrm{NO}$, y la forma oxidada o que pierde el grupo heme, asociada a condiciones del tipo estrés oxidativo e involucrada en el desarrollo de variadas enfermedades cardiovasculares. La forma libre del grupo heme ya no es sensible al NO y se denomina como disfuncional ${ }^{31}$.

El cGMP activa variados sistemas efectores, tales como la Proteína Quinasa dependiente de cGMP (PKG-1), que fosforila distintas proteínas blancos, que finalmente regulan de manera negativa la contracción mediada por la quinasa Rho A (ROCK-2), inhibiendo la acción de la vía independiente de calcio de la vasoconstricción ${ }^{32,33}$. ROCK-2 tiene un importante rol en la mediación de la vasoconstricción y el remodelamiento vascular en la patogénesis de la hipertensión pulmonar. ROCK induce la vasoconstricción mediante la fosforilación de la subunidad de la fosfatasa de la cadena liviana de la miosina, por lo tanto, disminuyendo la actividad fosfatásica y aumentando la contracción del músculo liso vascular ${ }^{3}$ (Figura 1).

Otro agonista vasodilatador de importancia en la circulación pulmonar, durante el período neonatal, es la prostaciclina. Esta juega un papel importante en la salud cardiovascular, específicamente modulando la vasodilatación a través de la relajación del músculo liso, al igual que la PKG-1, inhibe principalmente la ROCK quinasa, bloqueando la vía vasoconstrictora independiente de calcio ${ }^{34}$. La biosíntesis de la prostaciclina comienza con la liberación de ácido araquidónico (AA) desde la membrana plasmática por fosfolipasa $\mathrm{A}_{2}\left(\mathrm{PLA}_{2}\right)$, y posteriormente a través de la acción de ciclo-oxigenasas (COXs), catalizan la conversión de AA en prostaglandinas, dentro de las cuales se incluye prostaciclina (PGI2) y tromboxano $\left(\mathrm{TXA}_{2}\right)$. La síntesis de $\mathrm{PGI}_{2}$ está regulada por diferentes procesos intracelulares, incluyendo la fosforilación por quinasas de proteínas y la modificación de factores de transcripción nucleares ${ }^{34}$.

La prostaciclina ejerce su efecto a través de su receptor (IP), este está conformado por siete dominios transmembrana acoplado a proteína $\mathrm{G}$ estimuladora alfa ligante de guanosina $(\mathrm{G} \alpha \mathrm{s})^{35}$. 
Cuando IP es activado por $\mathrm{PGI}_{2}$, estimula a adenilato ciclasa $(\mathrm{AC})$, cuya isoforma más importante a nivel pulmonar es la $\mathrm{AC}_{2}$, incrementando los niveles intracelulares de adenosina-monofosfato cíclico (AMPc). Los niveles incrementados de AMPc conllevan a la activación de la proteína quinasa A (PKA) y posterior fosforilación de proteínas claves. Los efectos de la $\mathrm{PGI}_{2}$ en las células vasculares están mediados por diferentes vías de señalización, incluyendo la vía $\mathrm{PGI}_{2} / \mathrm{IP} /$ Gs/AMPc clásico y las vías intracrinas que implican receptores nucleares ${ }^{34}$.

\section{Agradecimientos}

Los autores agradecen el apoyo financiero a sus investigaciones otorgado por los proyectos FONDECyT 114064, 1151119 y VID Enlace Universidad de Chile.

\section{Bibliografía}

1.- RUDOLPH A. Fetal and Neonatal Pulmonary Circulation Ann. Rev. Physiol. 1979; 41: 383-95.

2.- ABMAN SH. Recent advances in the pathogenesis and treatment of persistent pulmonary hypertension of the newborn. Neonatology 2007; 91: 283-90.

3.- GAO Y, RAJ U. Regulation of the pulmonary circulation in the fetus and the newborn. Physiol Rev. 2010; 90: 12191-1335.

4.- HILLMAN N, KALLAPUR SG, JOBE A. Physiology of transition from intrauterine to extrauterine life. Clin Perinatol. 2012; 39: 769-83.

5.- HOOPER SB, TE PAS AB, LANG J, VAN VONDEREN JJ, ROEHR CC, KLUCKOW M, et al. Cardiovascular transition at birth: a physiological sequence. Pediatric Research. 2015; 77: 608-14.

6.- HAWORTH SG. Development of the normal and hypertensive pulmonary vasculature. Experimental physiology 1995; 80: 843-53.

7.- HAWORTH SG, HALL SM, CHEW M, ALLEN K. Thinning of fetal pulmonary arterial wall and postnatal remodeling: ultrastructural studies on the respiratory unit arteries of the pig. Virchows Arch A Pathol Anat Histopathol. 1987; 411: 161-71.

8.- PEÑALOZA D, ARIAS-STELLA J. The heart and pulmonary circulation at high altitudes: healthy highlanders and chronic Mountain sickness. Circulation 2007; 115: 1132-46.

9.- MURTHY KS. Signaling for contraction and relaxation in smooth muscle of the gut. Ann Rev Physiol. 2006; 68: 345-74.

10.- OGUT O, BROZOVICH F. The potential role of MLC phosphatase and MAPK signalling in the pathogenesis of vascular dysfunction in heart failure. J Cell Mol Med.
2008; 12: 2158-64.

11.- WRAY S , BURDYGA T. Sarcoplasmic reticulum function in smooth muscle. Physiological reviews. 2010; 90: 113-78.

12.- YANG XR, LIN MJ, SHAM JS. Physiological functions of transient receptor potential channels in pulmonary arterial smooth muscle cells. In: Membrane Receptors, Channels and Transporters in Pulmonary Circulation.Springer International Publishing AG. Humana Press. New York. 2010; pp 109-22.

13.- JERNIGAN NL, RESTA TC. Calcium homeostasis and sensitization in pulmonary arterial smooth muscle. Microcirculation. 2014; 21: 259-71.

14.- BUTLER T, PAUL J, EUROPE-FINNER N, SMITH R, CHAN EC. "Role of serine-threonine phosphoprotein phosphatases in smooth muscle contractility". Am J Physiol - Cell Physiol 2013; 304: C485-504.

15.- XIONG Z, SPERELAKIS N. Regulation of L-type calcium channels of vascular smooth muscle cells. J Molecular cellular cardiol. 1995; 27: 75-91.

16.- RAEYMAEKERS L, HOFMANN F, CASTEELS R. Cyclic GMP-dependent protein kinase phosphorylates phospholamban in isolated sarcoplasmic reticulum from cardiac and smooth muscle. Biochem J. 1988; 25226973.

17.- SYLVESTER JT, SHIMODA LA, AARONSON PI, WARD JPT. Hypoxic pulmonary vasoconstriction. Physiol Rev 2012; 92: 367-520.

18.- KHAPCHAEV AY, SHIRINSKY P. Myosin light chain kinase MYLK1: anatomy, interactions, functions, and regulation. Biochemistry (Moscow); 2016; 81: 1676-97.

19.- WILSON DP, SUSNJAR M, KISS E, SUTHERLAND C, WALSH MP. Thromboxane A2-induced contraction of rat caudal arterial smooth muscle involves activation of $\mathrm{Ca}^{2+}$ entry and $\mathrm{Ca}^{2+}$ sensitization: Rho-associated kinase-mediated phosphorylation of MYPT1 at Thr-855, but not Thr-697. Biochem J. 2005; 389: 763-74.

20.- KAWKA DW, OUELLET M, HÉTU P, SINGER II, RIENDEAU D. Double-label expression studies of prostacyclin synthase, thromboxane synthase and COX isoforms in normal aortic endothelium. Biochim Biophys Acta 2007; 1771: 45-54.

21.- DELANEY C, GIEN J, GROVER TR, ROE G, ABMAN SH. Pulmonary vascular effects of serotonin and selective serotonin reuptake inhibitors in the lategestation ovine fetus. American J Physiol-Lung Cellular Molecular Physiol. 2011, 301: L937-L944.

22.- KLOZA M, BARANOWSKA-KUCZKO M, PEDINSKA-BETIUK A, JACKOWSKI K, KOSLOWSKA H. Serotonin hypothesis and pulmonary artery hypertension. Postepy Hig Med Dows. 2014; 68: 738-48.

23.- WEBB RC. Smooth muscle contraction and relaxation. Advn Physiol education. 2003; 27: 201-16. 
24.- DENG M, DENG L, XUE Y. MAP Kinase-Mediated and MLCK-Independent Phosphorylation of MLC20 in Smooth Muscle Cells. From Molecules to Humans, Dr. Haruo Sugi (Ed.), In Tech, 2012. doi: 10.5772/47732.

25.- BOS C, RICHEL D, RITSEMA T, PEPPELENBOSCH M, VERSTEEG H. Prostanoids and Prostanoid Receptors in Signal Transduction. The International Journal of Biochemistry \& Cell Biology 2004; 36: 1187-205.

26.- DAFF S. NO synthase: Structures and mechanisms. Nitric Oxide-Biology and Chemistry. 2010; 23: 1-11.

27.- FLAVAHAN S, FLAVAHAN NA. The atypical structure and function of newborn arterial endothelium is mediated by Rho/Rho kinase signaling. Am J Physiol. Heart and circulatory physiol 2014; 307: H628-H632.

28.- GAO Y, CORNFIELD DN, STENMARK KR, THÉBAUD B, ABMAN SH, RAJ JU. Unique Aspects of the Developing Lung Circulation: Structural Development and Regulation of Vasomotor Tone 1. Pulmonary Circulation 2016; 6: 407-25.

29.- HERRERA EA, REYES VR, GUISSANI DA, RIQUELME RA, SANHUEZA EM, EBENSPERGER $\mathrm{G}$, et al. Carbon monoxide: a novel pulmonary artery vasodilator in neonatal llamas of the Andean altiplano.
Cardiovasc Res. 2008; 77: 197-201.

30.- RYTER SW, CHOI AMK. Targeting heme oxygenase-1 and carbon monoxide for therapeutic modulation of inflammation. Translational Research. 2016; 167: 7-34.

31.- DERBYSHIRE ER, MARLETTA MA. Biochemistry of soluble guanylate cyclase. Handbook of experimental pharmacology 2009; 191: 17-31.

32.- JERNIGAN NL, WALKER BR, RESTA TC. Reactive oxygen species mediate RhoA/Rho kinase-induced $\mathrm{Ca} 2+$ sensitization in pulmonary vascular smooth muscle following chronic hypoxia. Am J Physiol-Lung cellular and molecular physiology. 2008; 1: 515-29.

33.- VANHOUTTE PM, SHIMOKAWA H, FELETOU M, TANG EHC. Endothelial dysfunction and vascular disease - a 30th anniversary update. Acta Physiol. 2015; 219: 22-96.

34.- BATOULE M, RAOUF K. Molecular mechanisms regulating the vascular prostacyclin pathways and their adaptation during pregnancy and in the newborn. Pharmacol Rev 2012; 64: 540-82.

35.- STACY LD, STOKES P Jr. PGI2 as a regulator of inflammatory diseases. Mediators of Inflammation. 2012; 2012: 1-9.

Correspondencia a:

Germán Ebensperger Darrouy, PhD.

Profesor Asistente. Programa de Fisiopatología.

Instituto de Ciencias Biomédicas

Facultad de Medicina. Universidad de Chile.

Avda. Salvador 486, Providencia. Santiago de Chile.

Email: gebensperger@med.uchile.cl 